\title{
Flux Richardson Number and Turbulent Prandtl Number in a Developing Stable Boundary Layer
}

\author{
Yuji KITAMURA \\ Meteorological Research Institute, Japan Meteorological Agency \\ Akihiro HORI and Toshimasa YAGI \\ Meteorological and Environmental Sensing Technology, Inc. \\ (Manuscript received 28 January 2013, in final form 21 June 2013)
}

\begin{abstract}
Wind tunnel experiments and large-eddy simulations for stable stratification are performed to specify flux Richardson number Rf and turbulent Prandtl number Pr as a function of gradient Richardson number Ri. We attempted to avoid self-correlation by using independent samples for the variables commonly contained in these nondimensional numbers and confirmed the dependence of $\mathrm{Rf}$ and $\mathrm{Pr}$ on $\mathrm{Ri}$ for $10^{-3}<\mathrm{Ri}<5$. We found that $\mathrm{Rf}$ could exceed unity in a stable boundary layer under a developing stage, while the assumption of local energy balance violates for $\operatorname{Rf}>1$, which corresponds to negative production of turbulent kinetic energy. Nevertheless, the analysis of the TKE budget shows that the third-order term in the prognostic equation of TKE, which plays a role in the TKE transfer, can contribute to increase TKE despite negative TKE production. Therefore, TKE cannot be determined locally and the effects of TKE transfer must be taken into account in the region satisfying $\operatorname{Rf}>1$.
\end{abstract}

Keywords flux Richardson number; turbulent Prandtl number; stable boundary layer; turbulent kinetic energy budget

\section{Introduction}

Accurate estimation of second-order turbulent fluxes is essential for clarifying the atmospheric boundary layer, since mean velocity and temperature profiles are determined by divergence or convergence of the fluxes. In particular, understanding the effects of the static stability is important, which characterizes the structure of the atmospheric boundary layer. Flux Richardson number Rf and turbulent Prandtl number $\mathrm{Pr}$, as well as the scaling parameters in terms of the Monin-Obukhov similarity theory (e.g., Businger et al. 1971), have been widely regarded as the key parameters characterizing the turbulent flux (Derby-

Corresponding author: Yuji Kitamura, Meteorological Research Institute, Japan Meteorological Agency, 1-1 Nagamine, Tsukuba, Ibaraki 305-0052, Japan

E-mail: kitamura@mri-jma.go.jp

(C)2013, Meteorological Society of Japan shire 1999). Flux Richardson number is a stability parameter derived from momentum and heat fluxes. Turbulent Prandtl number is defined as the ratio of turbulent eddy diffusivities for heat and momentum, which could be also expressed as $\operatorname{Pr}=\mathrm{Ri} / \mathrm{Rf}$, where Ri is gradient Richardson number. Many studies have attempted to investigate dependence of these numbers on gradient Richardson number, which is derived from mean velocity and temperature gradients. In a Reynolds averaged numerical model (e.g., Mellor and Yamada 1982), Rf and Pr are unknown numbers that must be parameterized, while $\mathrm{Ri}$ can be explicitly diagnosed. Therefore, specifying $\mathrm{Rf}$ or $\mathrm{Pr}$ as a function of $\mathrm{Ri}$ is also practically important and provides useful information on validating turbulent parameterization.

However, measuring flux Richardson number and turbulent Prandtl number under strong stratification is difficult because of diminishing turbulent flux. Nevertheless, many researchers have attempted such 
measurements. Kondo et al. (1978) measured Rf through field observations of a stable surface layer and concluded that flux Richardson number had an upper limit, called the critical flux Richardson number $\mathrm{Rf}_{c}$, probably from 0.1 to 0.4 . Esau and Grachev (2007) analyzed their large-eddy simulation (LES) data and the Surface Heat Budget of the Arctic Ocean project data (Uttal et al. 2002) in comparison with those of the previous studies on the basis of field observations, wind tunnel experiments, and numerical simulations. They found that $\mathrm{Rf}_{c}$ was from 0.08 to 0.5 . Zilitinkevich et al. (2008) proposed an empirical formula, $\operatorname{Pr}=0.8+$ $5 \mathrm{Ri}$, on the basis of the analysis of the results of the previous studies. This formula corresponds that flux Richardson number approaches 0.2 at the $\mathrm{Ri} \rightarrow \infty$ limit. Strang and Fernando (2001) and Monti et al. (2002) reported that $\mathrm{Rf}(\mathrm{Ri})$ does not monotonically increase but has a peak, whose value is $\mathrm{Rf} \approx 0.4$ at $\mathrm{Ri}$ $\approx 1$. A common feature seen in the previous studies is that flux Richardson number in stable stratification has an upper limit less than unity, though its value still remains uncertain. Flux Richardson number less than unity means that the production term of turbulent kinetic energy (TKE) always has a positive value. This fact appears to justify the results of the previous studies because negative TKE production seldom gives a local equilibrium state of TKE.

The upper limit of flux Richardson number also relates to a critical gradient Richardson number at which turbulence ceases to occur in stable stratification. Whether a critical gradient Richardson number exists has been discussed for decades (e.g., Derbyshire 1999) and is still an open question. Most of the firstorder turbulent closure models have a critical value of gradient Richardson number, while some turbulent models that do not involve the critical gradient Richardson number have been proposed recently (Galperin et al. 2007; Mauritsen et al. 2007; Zilitinkevich et al. 2007; Canuto et al. 2008; Kitamura 2010a). As mentioned above, satisfying $\mathrm{Rf} \leq 1$ is necessary for a first order closure model assuming a local equilibrium of TKE. However, whether or not a closure model has a critical gradient Richardson number is sensitive to a maximum value of flux Richardson number; only the condition that Rf seldom exceeds unity may not be sufficient for a closure model without a critical gradient Richardson number. For instance, the modified MYNN level 2 model proposed by Kitamura (2010a) requires $\mathrm{Rf} \leq 0.298$ as the necessary condition for no critical gradient Richardson number. Hence, more accurate evaluation of the upper limit of flux Richardson number is necessary for exploring the issue of the critical gradient Richardson number.

Another problem in identifying the empirical relationship between $\mathrm{Rf}$ and $\mathrm{Ri}$ or between $\mathrm{Pr}$ and $\mathrm{Ri}$ is self-correlation, which arises from common variables between two parameters. For instance, the vertical shear of the mean velocity $d \bar{u} / d z$ is commonly included in the definitions of $\mathrm{Rf}$ and $\mathrm{Ri}$. Because observational error of the common variables results in spurious correlation, self-correlation prevents us from discerning a true correlation from a spurious one (Baas et al. 2006). Grachev et al. (2007) found that Pr increased with Ri in stable stratification, whereas it decreased with increasing Rf. They attributed this paradoxical behavior to self-correlation and proposed that $\mathrm{Pr}$ should be plotted against bulk Richardson number, which involves no shared variable. Anderson (2009) proposed indirect determination of $\operatorname{Pr}^{-1}(\mathrm{Ri})$ to avoid the effects of self-correlation. In this approach, the flux and velocity shear are first estimated as a function of the temperature gradient using the linear regression analysis for limited Ri sections, and then Pr and Ri are reconstructed from these regression coefficients at each Ri region.

In the present study, we perform wind tunnel experiments and numerical simulations using LES for stable stratification cases, and we attempt to identify flux Richardson number $\mathrm{Rf}$ and turbulent Prandtl number $\operatorname{Pr}$ as a function of gradient Richardson number Ri. We will focus on a stable boundary layer under a developing stage in the present analysis. In a steady state, the local balance in the TKE budget is required, which yields the restriction of $\mathrm{Rf} \leq 1$. On the other hand, it would be not necessary to theoretically satisfy $\mathrm{Rf} \leq 1$ in a developing boundary layer, because the time variation of the TKE is allowed in such a case. Investigating a boundary layer in a developing stage would be useful for validating an upper limit of Rf, which is suggested in the previous study. Wind tunnel experiments enables a direct and precise measurement for temperature and wind velocity. However, these variables are obliged to be observed as time series at a point in laboratory experiments. Hence, obtaining a great deal of data is difficult for an observational study. On the other hand, LES enables efficiently obtaining turbulent fields in various ideal environments and is a useful tool to determine the nondimensional parameters. However, LES data cannot be the alternative of observation because of uncertainty due to the effects of subgrid scale parameterization. We first compare the results obtained from LES with those obtained from wind 
tunnel experiments, and then estimate the profiles of $\mathrm{Rf}(\mathrm{Ri})$ and $\operatorname{Pr}^{-1}(\mathrm{Ri})$ from the LES data. As described above, this analysis requires a careful treatment to exclude a spurious relationship due to self-correlation. We attempt to directly avoid the effects of selfcorrelation by extracting two independent samples from obtained data. Our procedure for avoiding selfcorrelation will be described in Section 3.

This paper is organized as follows. Section 2 describes the methods of wind tunnel experiments and numerical simulations using LES. Section 3 is devoted to the data analysis procedure. The first part of Section 4 discusses the dependence of $\mathrm{Rf}$ and $\mathrm{Pr}$ on $\mathrm{Ri}$. The second part of Section 4 explores the TKE budget on the basis of the results of LES. Section 5 presents our concluding remarks.

\section{Experimental methods}

\subsection{Wind tunnel experiment}

The wind tunnel experiments were originally performed in a wind tunnel at the Meteorological Research Institute. It has an 18-m streamwise section, a 3-m spanwise section, and a 2-m floor-normal section. The wind tunnel is capable of controlling the temperature on the floor in the test section. A stable boundary layer can be generated by lowering the floor temperature. Throughout the experiments, 3-mm diameter round bars were placed at intervals of $10 \mathrm{~cm}$ on the floor as surface roughness for the boundary layer.

We performed a series of experiments with the freestream velocity $U_{\infty}=2,4,6$, and $8 \mathrm{~m} \mathrm{~s}^{-1}$. The floor temperature was kept $10 \mathrm{~K}$ cooler than the ambient air to produce a stable boundary layer. Measurements were performed at $13.55 \mathrm{~m}$ from the entrance of the test section. In the present study, we first estimated the depth of the stable boundary layer from the preliminary experiments for each $U_{\infty}$. The vertical profiles of the streamwise momentum flux and temperature flux obtained from the preliminary experiments are shown in Fig. 1. The depth of the boundary layer was defined as the height at which these fluxes is less than $1 \%$ of the maximum values. It increased with larger $U_{\infty}$ and was approximately $28 \mathrm{~cm}$ and $40 \mathrm{~cm}$ for $U_{\infty}=2 \mathrm{~m} \mathrm{~s}^{-1}$ and $8 \mathrm{~m} \mathrm{~s}^{-1}$, respectively. Then, the velocity and temperature were simultaneously measured at intervals of $10 \mathrm{~mm}$ in the vertical direction in the upper region of the boundary layer, as larger $\mathrm{Ri}$, which is our interest, was realized in the top of the boundary layer because of monotonous increase of $\mathrm{Ri}$ with height. The velocity components in the streamwise and vertical directions $u$ and $w$, respective-
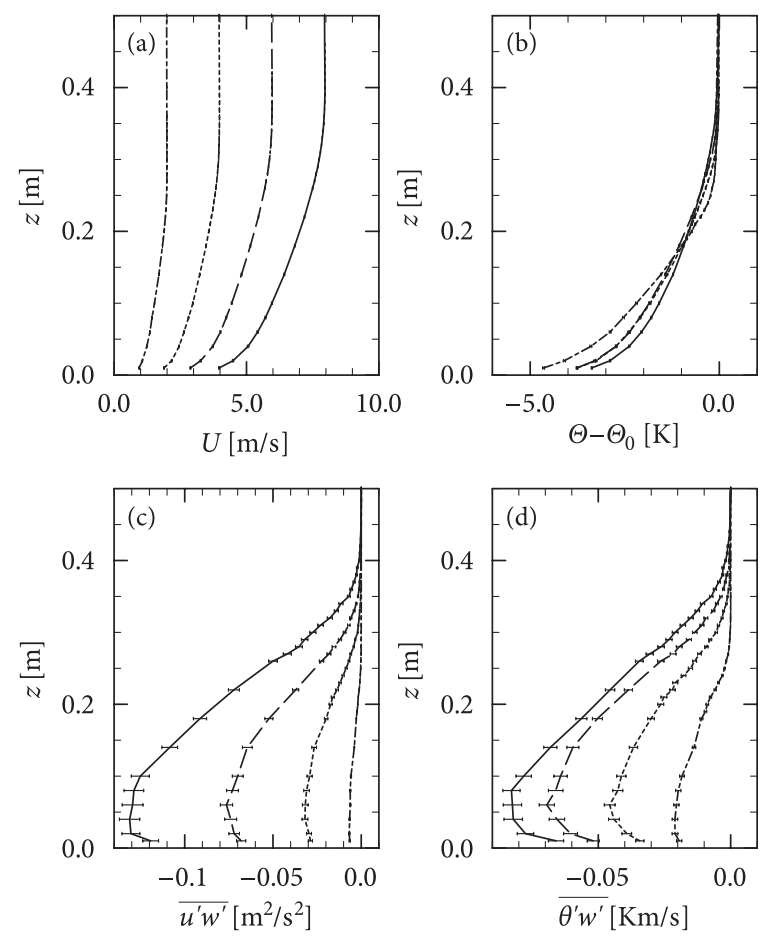

Fig. 1. Vertical profiles of (a) mean velocity in the streamwise direction, (b) difference of the mean temperature from the ambient air temperature, (c) momentum flux in the streamwise direction and (d) temperature flux obtained from the wind tunnel experiments. The solid, dashed, dotted, and dash-dotted lines correspond to $U_{\infty}=8,6,4$, and $2 \mathrm{~m} \mathrm{~s}^{-1}$, respectively. The error bar represents two times the standard deviation.

ly, were measured using laser doppler velocimetry (LDV, Dantec Dynamics FiberFlow 2D System), and the temperature was measured using an I-type coldwire probe with a $1 \mu \mathrm{m}$-diameter, $0.4 \mathrm{~mm}$-long platinum sensor. The temperature fluctuation signals were digitized at $100 \mathrm{~Hz}$. On the other hand, the LDV signals were obtained at irregular time intervals, although the sampling rate was confirmed to be a few times greater than $100 \mathrm{~Hz}$ throughout the experiments. The LDV signals were resampled to the data at 0.01 -s intervals to synchronize with the temperature time series. The sampling period was $300 \mathrm{~s}$. The vertical profiles of the velocity and temperature in the upper region of the boundary layer were measured six times for each $U_{\infty}$. Although the measurements were not performed simultaneously at the vertical points, each measurement can be regarded as a sample obtained from the same condition, since a boundary layer was 
spatially steady in the test section.

It should be noted that there are some uncertainties in the present measurement. Tsuji et al. (1992) reported the delay of the temperature fluctuations in lowfrequency regions due to the thermal inertia of a coldwire probe. Furthermore, the velocity and temperature cannot be measured at the exact same location, because disturbance of the flow field by the probe cannot be neglected in the vicinity of the probe. For this reason, the sampling points of the velocity and temperature are separated slightly (approximately $1 \mathrm{~mm}$ ). Although these factors could cause an underestimation of the temperature flux, we confirmed that the lagged covariance between the velocity and temperature fluctuations had a peak when the time lag was zero with a precision of $0.01 \mathrm{~s}$.

\subsection{LES}

The numerical simulation was performed with the following setup. The domain size in the numerical model was set at $256 \mathrm{~cm} \times 256 \mathrm{~cm}$ in the horizontal plane and $128 \mathrm{~cm}$ in the vertical direction, with a model resolution of $1 \mathrm{~cm}$. The lateral boundary condition was cyclic, and the free slip boundary condition was imposed at the top. The momentum and heat fluxes on the ground surface were diagnosed on the basis of the formulation by Louis et al. (1982). The roughness lengths were set to $z_{0 m}=10^{-4} \mathrm{~m}$ for the momentum and $z_{0 h}=10^{-5} \mathrm{~m}$ for the heat, which were determined to be close to those estimated from the wind tunnel experiment in the present study. However, this model setup is not completely identical to the environment realized in the wind tunnel experiment, especially for the lateral boundary condition, since the inflow is quite different from the outflow in the wind tunnel. Appropriately representing the inflow and outflow observed in the wind tunnel as a lateral boundary condition in the LES is difficult. Nevertheless, this does not prevent comparing the nondimensional numbers Ri, Rf, and Pr, which are focused in this study. We confirmed that the domain size used in the present experiments did not affect the results in comparison with the preliminary experiments using a wider computational domain.

The governing equations and subgrid scale (SGS) parameterization schemes used in the present study are same as those described in Kitamura (2010b). A nonhydrostatic incompressible Boussinesq fluid is assumed in the numerical model. A pseudospectral method was adopted for a horizontal plane, and the vertical discretization followed the Lorentz grid, in which the horizontal velocity components, potential temperature, and pressure are located at integer grids, and the vertical velocity at half-integer grids. The vertical differentiation was evaluated with a secondorder centered difference. Time integration was performed with the fourth-order Runge-Kutta method. Since the results obtained from LES could depend on a parameterization scheme to represent the effects of the SGSs, we examined two types of SGS models, the dynamic Smagorinsky model (Germano et al. 1991; Lilly 1992) and the Deardorff model (Deardorff 1980), and confirmed that the statistical properties obtained in the present experiments did not depend on these SGS models.

In contrast with the wind tunnel experiments in which the temperature of the incoming flow was uniform in height, stable stratification in the initial potential temperature profile was imposed in the numerical simulations to efficiently create a flow field with larger Ri. In the present study, the horizontal mean of initial potential temperature increases linearly with height: $\Theta(z)=288.15+\Gamma z \mathrm{~K}$, where $\Gamma$ indicates the rate of temperature increase with height. The ground temperature was set at $278.15 \mathrm{~K}$, which is $10 \mathrm{~K}$ cooler than the initial air temperature at the bottom as well as in the wind tunnel experiments. Initial perturbation of random noise was added to the potential temperature field with an amplitude of 0.02 $\mathrm{K}$. On the other hand, the initial velocity field was uniform throughout the whole domain. We examined two static stabilities $\Gamma=8$ and $16 \mathrm{~K} \mathrm{~m}^{-1}$ and three initial velocities, $U=2,4$, and $8 \mathrm{~m} \mathrm{~s}^{-1}$. Time integration was halted at 40,20, and $10 \mathrm{~s}$ for $U=2,4$, and $8 \mathrm{~m} \mathrm{~s}^{-1}$, respectively. The turbulent field was under a developing stage throughout these time integration periods, although the momentum will be extinct after a long run because of no external forcing. Moreover, the ensemble experiment with ten members, for which only initial perturbation differs, was conducted for each initial mean profile and each SGS parameterization scheme, and the nondimensional numbers were calculated from each ensemble member, since increasing the number of the samples contributes to more reliable estimation of $\mathrm{Rf}$ and $\mathrm{Pr}$.

\section{Analysis procedure}

First, the variables $u, w$, and $\theta$ were divided into an average and its deviation as follows:

$$
u=\bar{u}+u^{\prime}, \quad w=\bar{w}+w^{\prime}, \quad \theta=\bar{\theta}+\theta^{\prime} .
$$

Here $u$ and $w$ are the velocity components of the streamwise and vertical directions, respectively, and $\theta$ is the potential temperature. The overbar denotes an 
average, and the prime denotes a deviation from the average. The average was defined as the time average of each time series for the wind tunnel experiment and the spatial average over a horizontal plane for the numerical simulation. It should be noted that different averages are considered for the wind tunnel experiment and LES. In the wind tunnel experiment, the flow field spatially develops toward the downstream direction, and the statistical features of turbulence at a point should be temporally fixed. Therefore, a temporal average at a point is suitable for the wind tunnel experiment. On the other hand, the boundary layer in the LES develops uniformly in a horizontal plane with time evolution, because the lateral boundary condition was cyclic in our configuration. So, a spatial average is preferable for the LES data. Although these statistics could not be identical, one can expect that the nondimensional numbers $\mathrm{Rf}$ and $\mathrm{Pr}$ possess a universal dependence. However, this expectation has to be confirmed in the comparison between the results obtained from the wind tunnel experiment and those obtained from the LES.

Gradient Richardson number Ri, flux Richardson number Rf, and turbulent Prandtl number Pr are defined as

$$
\begin{aligned}
& \operatorname{Ri}=\frac{g}{\bar{\theta}} \frac{d \bar{\theta} / d z}{(d \bar{u} / d z)^{2}}, \quad \operatorname{Rf}=\frac{g}{\bar{\theta}} \frac{\overline{\theta^{\prime} w^{\prime}}}{\overline{u^{\prime} w^{\prime}}(d \bar{u} / d z)}, \\
& \operatorname{Pr}=\frac{\overline{u^{\prime} w^{\prime}} d \bar{\theta} / d z}{\overline{\theta^{\prime} w^{\prime}} d \bar{u} / d z},
\end{aligned}
$$

where $g$ denotes the acceleration of gravity. In the following, we consider $\operatorname{Pr}^{-1}$ instead of $\operatorname{Pr}$ itself, following Anderson (2009).

For the purpose of data quality control, the analysis was limited to only the data satisfying $d \bar{u} / d z>0, d \bar{\theta} / d z$ $>0, \overline{u^{\prime} w^{\prime}}<0$ and $\overline{\theta^{\prime} w^{\prime}}<0$. These limitations correspond that the momentum and heat are transferred to the ground surface and these fluxes are directed downgradient. Furthermore, the region where the SGS flux exceeds $10 \%$ of the total flux or the total flux is below $1 \%$ of the surface flux was excluded from the analysis for the LES data, to reduce uncertainty of the parameterized SGS flux.

For the experimental data, errors of $\mathrm{Ri}, \mathrm{Rf}$, and $\mathrm{Pr}^{-1}$ were estimated on the basis of the standard deviation of the variables, $d \bar{u} / d z, d \bar{\theta} / d z, \overline{u^{\prime} w^{\prime}}$, and $\overline{\theta^{\prime} w^{\prime}}$. The variation of $\bar{\theta}$ itself was not considered, because it is much smaller than that of other variables. It should be noted that the degree of freedom, which is necessary to calculate a standard deviation for average, cannot be regarded as the number of samples in a time series, because samples included in a time series are not independent of each other. The effective number of independent samples was estimated in terms of the autocorrelation time scale presented by Metz (1991). Using the effective number of samples, we calculated the standard deviations $\sigma_{u_{z}}, \sigma_{\theta_{z}}, \sigma_{u w}$, and $\sigma_{\theta_{w}}$ and then estimated the errors of the nondimensional numbers on the basis of the propagation of errors as

$$
\frac{\sigma_{\mathrm{Ri}}}{\mathrm{Ri}}=\left[\left(\frac{2 \sigma_{u_{z}}}{d \bar{u} / d z}\right)^{2}+\left(\frac{\sigma_{\theta_{z}}}{d \bar{\theta} / d z}\right)^{2}\right]^{1 / 2} .
$$

Here $\sigma_{\mathrm{Ri}}$ denotes the standard deviation for the estimate of Ri.

The LES data obtained in the latter half of the integration period was analyzed in terms of the conditional probability given $\mathrm{Ri}$, rather than estimation of the standard deviation for each sample. We obtained 500 snapshots for each numerical experiment and calculated the vertical profile of $\mathrm{Ri}, \mathrm{Rf}$, and $\mathrm{Pr}^{-1}$ from the horizontal average of the variables for each snapshot. We first calculated the conditional probability density functions of $P(\mathrm{Rf} \mid \mathrm{Ri})$ and $P\left(\mathrm{Pr}^{-1} \mid \mathrm{Ri}\right) . \mathrm{Rf}(\mathrm{Ri})$ and $\operatorname{Pr}^{-1}(\mathrm{Ri})$ are estimated as a function of Ri from the expected values of $\log (\mathrm{Rf})$ and $\log \left(\mathrm{Pr}^{-1}\right)$, respectively. It should be noted that the expected values of $\mathrm{Rf}$ and $\mathrm{Pr}^{-1}$ tend to be much greater than these median values especially in the high $\mathrm{Ri}$ region, because these probability density functions have highly skewed distributions (Esau and Grachev 2007). The expected value calculated by the logarithmic value was confirmed to be close to the median. For this reason, the logarithmic values were adopted in the present analysis.

As described in the Introduction, reducing the effects of self-correlation is necessary for accurately estimating $\mathrm{Rf}(\mathrm{Ri})$ and $\operatorname{Pr}^{-1}(\mathrm{Ri})$. Equation (1) indicates that Rf and Ri share $d \bar{u} / d z$, and $\mathrm{Rf}$ and $\operatorname{Pr}^{-1}$ have the common variables $d \bar{u} / d z$ and $d \bar{\theta} / d z$. Spurious correlation involved with the error of these shared variables can be vanished, if independent samples are used in calculating $\mathrm{Ri}$ and $\mathrm{Rf}$ or $\mathrm{Pr}^{-1}$. In the present study, independent data for the shared variables were made by a time series of the wind tunnel data divided into two time series for which each sampling period is 150 s. These two samples obtained by this procedure could be regarded as independent ones extracted from the same environment, because the autocorrelation time for these time series is much shorter than the sampling period. Then, Rf or $\operatorname{Pr}^{-1}$ was calculated using a different time series from that used in the calculation of $\mathrm{Ri}$, to exclude spurious correlation due to the errors included in the shared variables. Two independent samples for the LES data were created by dividing a 

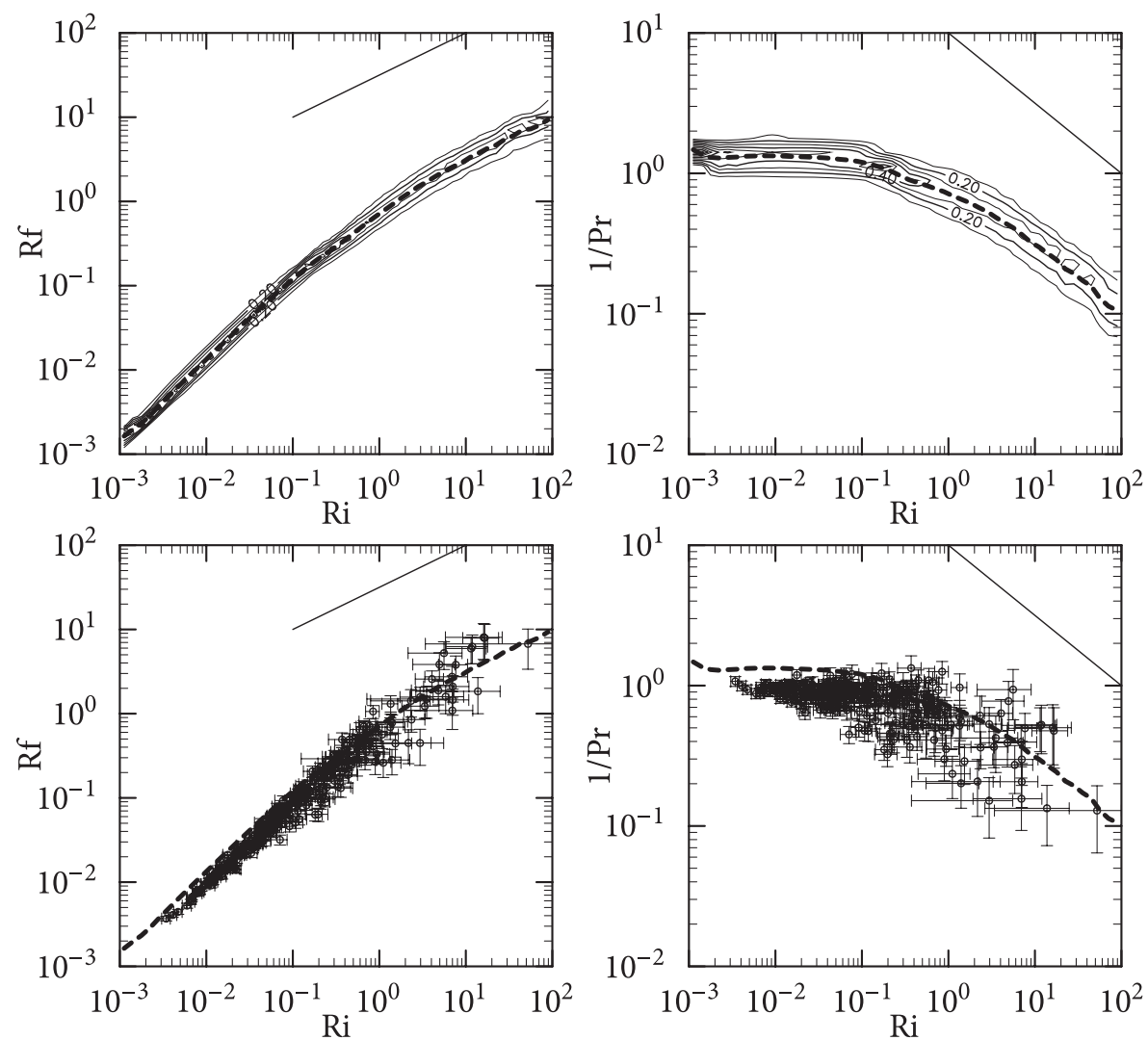

Fig. 2. Flux Richardson number Rf (left) and the inverse of turbulent Prandtl number $\operatorname{Pr}^{-1}$ (right) versus gradient Richardson number Ri. The upper panels indicate the conditional probability density given Ri obtained from the LES, and the lower panels indicate scatter plots with error bars obtained from the wind tunnel experiments. The error bar represents one standard deviation. The thick lines indicate the expected values of $\log (\mathrm{R} f) \operatorname{and} \log \left(\operatorname{Pr}^{-1}\right)$ estimated from the LES data. The reference lines represent $\mathrm{Ri}^{1 / 2}$ (left panels) and $\mathrm{Ri}^{-1 / 2}$ (right panels). In this figure, independence of samples in the shared variables is not considered, and the effects of self-correlation are included.

horizontal plane into two areas and averaging over each area.

\section{Results}

\subsection{Dependence of $R f$ and $\mathrm{Pr}^{-1}$ on $\mathrm{Ri}$}

Figure 2 presents Flux Richardson number Rf and the inverse of turbulent Prandtl number $\operatorname{Pr}^{-1}$. Independence of the samples in the shared variables is not considered here. Both Rf and $\operatorname{Pr}^{-1}$ appear to have universal dependence on Ri. The results obtained from the LES are consistent with those of the wind tunnel experiments, although $\operatorname{Pr}^{-1}$ observed in the wind tunnel experiments is slightly smaller than that in the LES in the small Ri region. $\mathrm{Rf}(\mathrm{Ri})$ increases linearly as $10^{-3}<\mathrm{Ri}<1$. In contrast to the previous studies, $\mathrm{Rf}(\mathrm{Ri})$ does not converge to a value less than unity, although its slope becomes less for $\mathrm{Ri}>1 . \mathrm{Pr}^{-1}(\mathrm{Ri})$ is almost constant for $\mathrm{Ri}<0.2$, whose value is close to unity in the wind tunnel experiments and approximatey 1.4 in the LES, and it monotonically decreases for $\mathrm{Ri}>0.2$. The definition of the nondimensional numbers Eq. (1) suggests that the self-correlation is prone to cause the relationships $\mathrm{Rf} \propto \mathrm{Ri}^{1 / 2}$ and $\operatorname{Pr}^{-1} \propto$ $\mathrm{Ri}^{-1 / 2}$. The result observed in Fig. 2 appears to be contaminated by the self-correlation for Ri $>1$.

Following the procedure described in Section 3, we analyze the dependence of $\mathrm{Rf}$ and $\mathrm{Pr}^{-1}$ on $\mathrm{Ri}$ using independent samples for the shared variables, to clarify the effects of self-correlation (Fig. 3). The results of the LES and wind tunnel experiments remain consistent, while uncertainty of the results obtained from the wind tunnel experiments increases in the large Ri region. The large scatter attributed that the vertical shear $d \bar{u} / d z$, which is included in the denominator of the 

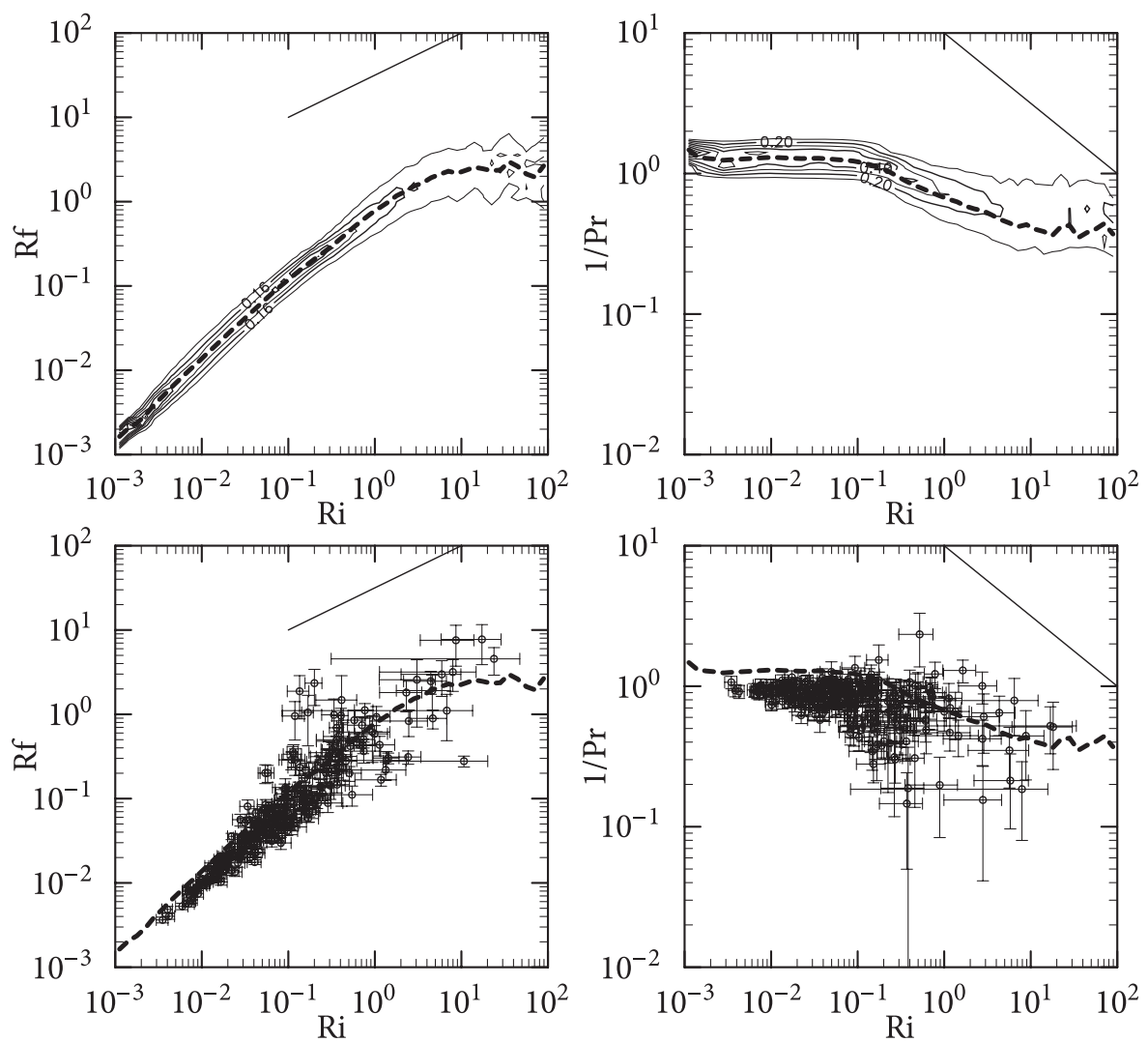

Fig. 3. Same as Fig. 2, except that independent samples are used in calculating Ri and Rf or Ri and $\operatorname{Pr}^{-1}$, following the procedures described in Section 3. Built-in correlation is excluded in this figure.

nondimensional numbers (Eq. 1), decreases under the strongly stable condition. The estimate is remarkably sensitive to the error of the denominator when the mean value of the denominator is close to zero. Reducing this type of error is extremely difficult. The consistency between the wind tunnel and LES results suggests that these nondimensional numbers possess a universal dependence even though the time average in a time series at one point obtained from the wind tunnel experiments could have a different meaning from the spatial average in the LES results. The effect of self-correlation remarkably appears in $\mathrm{Ri}>1$; the dependence of $\mathrm{Rf}$ and $\mathrm{Pr}^{-1}$ is quite different from that observed in Fig. 2. Although Rf tends to converge, Rf still exceeds unity in both the LES and wind tunnel experiments. $\operatorname{Pr}^{-1}$ decreases with $\mathrm{Ri}$ for $0.2<\mathrm{Ri}<10$, but its slope is shallower than that seen in Fig. 2 and becomes constant for $\mathrm{Ri}>10$.

Whether the results in Fig. 3 satisfy the theoretical expression $\operatorname{Pr}^{-1}=\mathrm{Rf} / \mathrm{Ri}$ is useful for validating the appropriateness of the empirical relationship obtained in the present experiment. Figure 4 indicates $\mathrm{Rf}(\mathrm{Ri})$ and $\mathrm{Ri} / \mathrm{Pr}(\mathrm{Ri})$ (left panel) and $\operatorname{Pr}^{-1}(\mathrm{Ri})$ and $\mathrm{Rf}(\mathrm{Ri}) / \mathrm{Ri}$ (right panel) estimated as the expected values of $\log (\mathrm{Rf})$ and $\log \left(\operatorname{Pr}^{-1}\right)$ in the LES data. $\operatorname{Pr}^{-1}(\mathrm{Ri})$ and $\mathrm{Rf}(\mathrm{Ri}) / \mathrm{Ri}$ coincide when $10^{-3}<\mathrm{Ri}<5$, and the robustness of the empirical relationship can be confirmed in this range. However, they are quite different for $\mathrm{Ri}>5$, where $\mathrm{Rf}(\mathrm{Ri})$ and $\operatorname{Pr}^{-1}(\mathrm{Ri})$ are almost constant. Inconsistency in Ri $>5$ might be attributed to an error resulting from overestimation of $\mathrm{Ri}$ in this range. Nevertheless, $\mathrm{Rf}$ exceeding unity is realized for $1<\mathrm{Ri}<5$ in the present experiments. Although turbulence tends to be intermittent in the region where $\mathrm{Rf}$ exceeds unity, turbulent flux still remains on average and a critical Richardson number cannot be observed when $\mathrm{Ri}<5$. It should be noted that $\mathrm{Rf}(\mathrm{Ri}) / \mathrm{Ri}$ and $\operatorname{Pr}^{-1}(\mathrm{Ri})$ coincide because of the spurious correlation, if self-correlation is not avoided. However, this coincidence does not assure appropriateness of the results. Avoiding self-correlation allows us to validate the results, even though the estimation of 

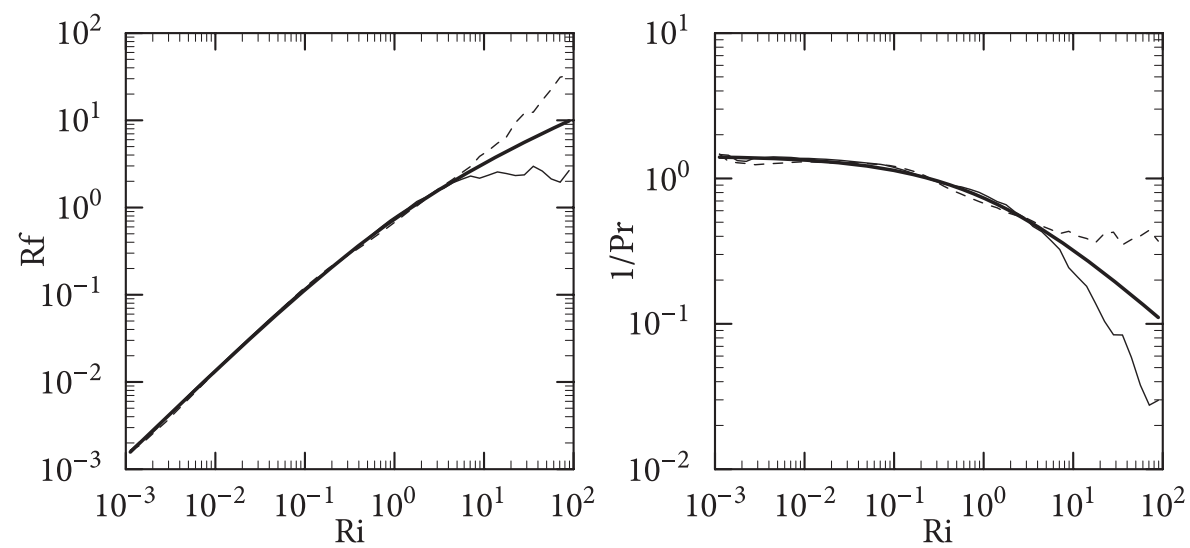

Fig. 4. Rf and $\operatorname{Pr}^{-1}$ as a function of Ri estimated by the expected values of $\log (\mathrm{Rf})$ and $\log \left(\operatorname{Pr}^{-1}\right)$ in the LES data. The thin solid and dashed lines indicate $\mathrm{Rf}(\mathrm{Ri})$ and $\mathrm{Ri} / \mathrm{Pr}(\mathrm{Ri})$ in the left panel and $\mathrm{Rf}(\mathrm{Ri}) / \mathrm{Ri}$ and $\operatorname{Pr}^{-1}(\mathrm{Ri})$ in the right panel. The thick line denotes the empirical function fitted by the least squares method when $10^{-3}<\mathrm{Ri}<5$. $\mathrm{Rf}(\mathrm{Ri})$ and $\operatorname{Pr}^{-1}(\mathrm{Ri})$ are calculated with independent samples as indicated in Fig. 3. Note that these two lines in each panel should be theoretically identical.

$\mathrm{Rf}(\mathrm{Ri})$ and $\operatorname{Pr}^{-1}(\mathrm{Ri})$ is erroneous. It is a distinct advantage in the present analysis.

Following Anderson (2009), we attempted to fit the Butterworth function

$$
\operatorname{Pr}^{-1}=\frac{\operatorname{Pr}_{0}^{-1}}{1+\left(\mathrm{Ri}_{2} / \mathrm{Ri}_{\mathrm{th}}\right)^{N}},
$$

to $\operatorname{Pr}^{-1}(\mathrm{Ri})$ and $\mathrm{Rf}(\mathrm{Ri}) / \mathrm{Ri}$ obtained from the LES data in the range of $10^{-3}<\mathrm{Ri}<5$, in which the robustness of the empirical relationship is confirmed. Here $\mathrm{Pr}_{0}{ }^{-1}$ corresponds to the inverse of turbulent Prandtl number in neutral stratification $(\mathrm{Ri}=0)$ and $\mathrm{Ri}_{\text {th }}$ characterizes the threshold over which $\operatorname{Pr}^{-1}$ tends to decrease with an increase of Ri. The regression based on the least squares method yields the values of the regression coefficients as $\left(\operatorname{Pr}_{0}^{-1}, \mathrm{Ri}_{\text {th }}, N\right)=(1.43,1.11,0.564)$. This fitted function is presented as the thick line in Fig. 4. The difference between the best fit Butterworth function and $\operatorname{Pr}^{-1}(\mathrm{Ri})$ estimated from the LES data is from $-6.8 \%$ to $+11.7 \%$ for $10^{-3}<\mathrm{Ri}<5$.

\subsection{Kinetic energy budget}

The results in the previous subsection show that flux Richardson number $\mathrm{Rf}$ can exceed unity in strong stratification. This means that the production of TKE becomes negative. Therefore, the local balance between the TKE production and dissipation cannot be realized when $\mathrm{Rf}>1$, while the local TKE balance is often assumed in the simplest first-order closure model. Clarifying the process which controls TKE is important, if the local balance of TKE cannot be allowed in a closure model. In this subsection, we analyze the TKE budget in the LES to discuss the TKE balance in the region with negative TKE production. In the following, only the results for a $U=4 \mathrm{~m} \mathrm{~s}^{-1}, \Gamma=8$ $\mathrm{K} \mathrm{m}^{-1}$ case simulated with the Deardorff model will be presented. Qualitative features associated with the TKE budget were confirmed to be insensitive to $U, \Gamma$, or an SGS model.

In the present analysis, the TKE $e$ is defined as that of a grid scale resolved explicitly. Although the subgrid part of the TKE, which is parameterized and remains uncertain, is not taken into account, this assumption would be reasonable, because the region where the SGS flux exceeds $10 \%$ of the total flux is excluded from the analysis. The prognostic equation of TKE averaged over a horizontal plane is described as

$$
\begin{aligned}
\frac{\partial \bar{e}}{\partial t}= & -\overline{u^{\prime} w^{\prime}} \frac{\partial \bar{u}}{\partial z}+\frac{g}{\bar{\theta}} \overline{\theta^{\prime} w^{\prime}}-\frac{\partial}{\partial z}\left(\overline{e w^{\prime}}+\overline{p^{\prime} w^{\prime}}\right) \\
& -\overline{u_{i}^{\prime} \frac{\partial\left(\tau_{i j}-\overline{\tau_{i j}}\right)}{\partial x_{j}}+\nu \overline{u_{i}^{\prime}} \frac{\partial^{2} u_{i}^{\prime}}{\partial x_{j} \partial x_{j}},}
\end{aligned}
$$

where $\tau_{i j}, \nu$, and $p$ denote the subgrid stress parameterized in the LES, molecular viscosity coefficient, and the pressure divided by the reference density, respectively. On the right-hand side, the first term represents shear production, and the second term represents buoyancy production. Note that these production terms can be rewritten as

$$
-\overline{u^{\prime} w^{\prime}} \frac{\partial \bar{u}}{\partial z}+\frac{g}{\bar{\theta}} \overline{\theta^{\prime} w^{\prime}}=-\overline{u^{\prime} w^{\prime}} \frac{\partial \bar{u}}{\partial z}(1-\mathrm{Rf}) .
$$



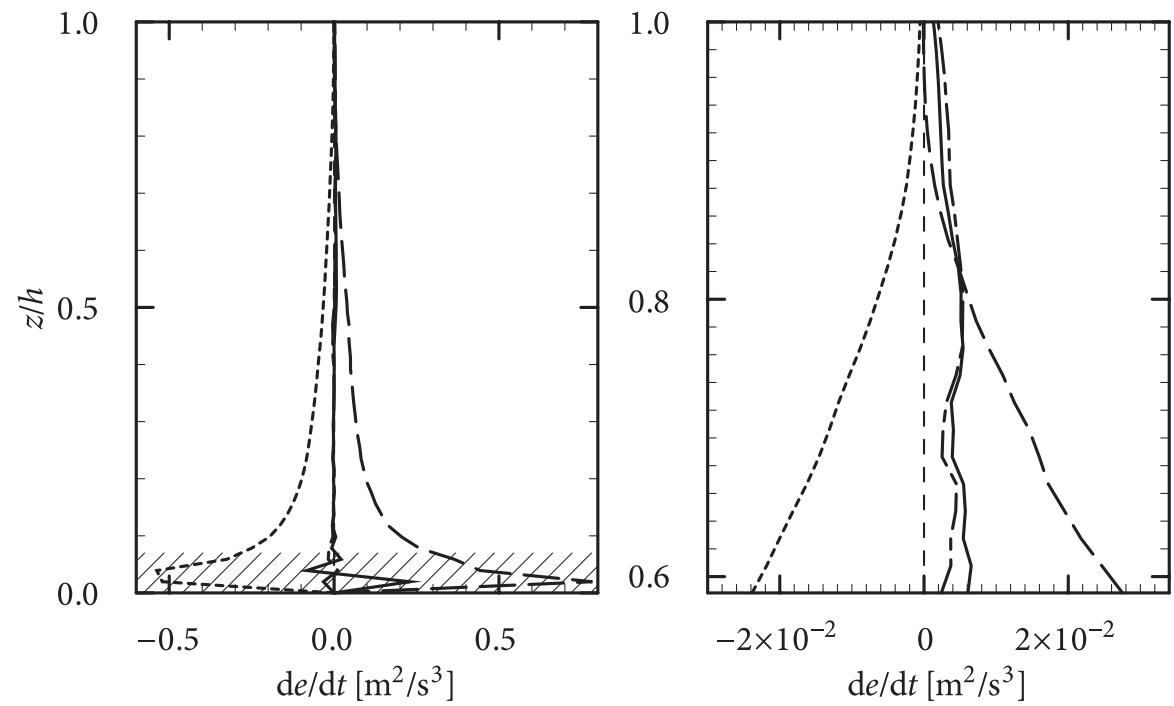

Fig. 5. Vertical profile of the TKE budget averaged over $t=9.5-10.5 \mathrm{~s}$ for a $U=4 \mathrm{~m} \mathrm{~s}^{-1}, \Gamma=8 \mathrm{~K} \mathrm{~m}^{-1}$ case simulated with the Deardorff model. The vertical scale is normalized by the height of the boundary layer $h$. The solid, dashed, dotted, and dash-dotted lines correspond to the total budget, production term, dissipation term, and transport term associated with the pressure and TKE, respectively. A profile of the entire boundary layer is presented in the left panel, and the right panel presents details in the upper region. The hatched area indicates the region where the conditions for the quality check described in Section 3 are not satisfied.

Since the results discussed in the previous section are derived from only the data satisfying $d \bar{u} / d z>0$ and $\overline{u^{\prime} w^{\prime}}<0$, Eq. (5) indicates that the sign of $(1-\mathrm{Rf})$ coincides with that of the production term. The third term represents the vertical transport of the TKE. The fourth and fifth terms correspond to TKE dissipation.

Figure 5 depicts a vertical profile of the TKE budget averaged over $t=9.5-10.5 \mathrm{~s}$ for a $U=4 \mathrm{~m} \mathrm{~s}^{-1}, \Gamma=8$ $\mathrm{K} \mathrm{m}^{-1}$ case simulated with the Deardorff model. In this figure, the height is normalized by the boundary layer height $h$ at which the momentum flux falls to $1 \%$ of the surface flux. Although the boundary layer height is defined as an averaged value for $t=9.5-10.5 \mathrm{~s}$ rather than an instantaneous one, it is confirmed that the variation of the height in this period is from $-2 \%$ to $4 \%$ and the time average does not essentially affect the normalized vertical profile of the TKE budget.

The production and dissipation terms are dominant and cancel each other when $z / h<0.7$. Hence, the assumption of local energy balance seems reasonable in this region. However, the magnitude of these terms decreases with height, while the transfer term does not almost change in magnitude. As a result, contribution of the TKE transfer becomes major in the top of the boundary layer $(z / h>0.8)$, and the TKE balance between production and dissipation has only minor importance for the TKE budget. This figure suggests that the TKE can increase because of a positive transfer to the top of the boundary layer, even though $\mathrm{Rf}>1$ corresponding to negative production. This result would be reasonable in a developing boundary layer; TKE is never supplied and a boundary layer no longer develops without a positive TKE transfer in the region of $\mathrm{Rf}>1$. The predominance of the transfer term in the top of the boundary layer can be always observed in the latter half of the time integration, for which the nondimensional numbers were analyzed in the previous section.

The temporal evolution of the TKE budget derived from each term is presented in Fig. 6. In this figure, the stable boundary layer develops and its depth increases with time. The production and dissipation terms almost cancel over the entire boundary layer, and the net TKE budget is determined mostly by the transfer term, of which the effect appears to propagate upward. Temporal variation of the transfer term is complicated, while the transfer term is always positive in the region where the production term is negative (hatched region). This positive TKE transfer increases TKE in the top of the boundary layer and contributes to development of the boundary layer.

The TKE transfer term consists of the convergences 

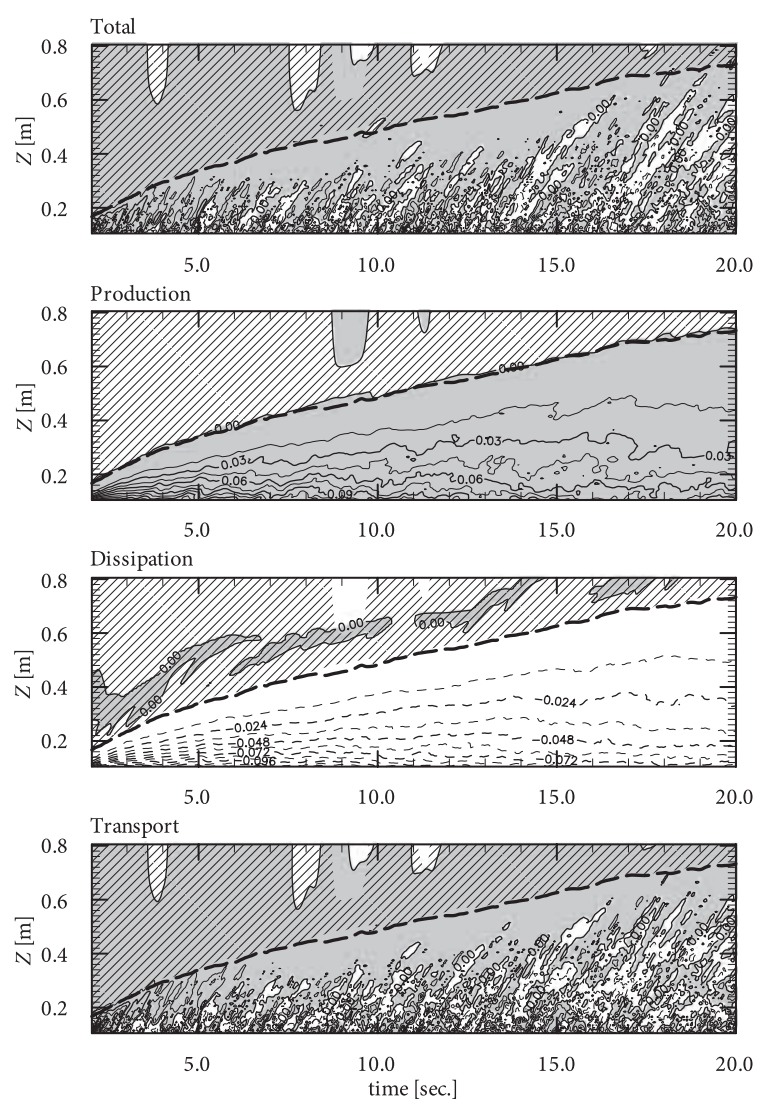

Fig. 6. Temporal evolution of the TKE budget for a $U=4$ $\mathrm{m} \mathrm{s}^{-1}, \Gamma=8 \mathrm{~K} \mathrm{~m}^{-1}$ case (same as Fig. 5). In this figure, the vertical profiles are drawn from instantaneous snapshots in contrast to Fig. 5. The gray area corresponds to a positive value. The hatched area indicates the region where the production term is negative $(\mathrm{Rf}>1)$. The thick dashed line indicates $\mathrm{Ri}=1$. In the area above this line, $\mathrm{Ri}$ always exceeds unity.

of the TKE flux $-\partial \overline{e w^{\prime}} / \partial z$ and pressure-velocity correlation $-\partial \overline{p^{\prime} w^{\prime}} / \partial z$ as indicated in Eq. (4). The vertical profile of these terms presented in Fig. 7 indicates that these terms have an obvious negative correlation in the whole boundary layer. Costa et al. (2011) pointed out that they had opposite signs near the surface in LES for a stable boundary layer presented in Kosović and Curry (2000). The pressure-velocity correlation term weakens the contribution of the TKE flux; however, its magnitude is smaller than that of the TKE flux. Consequently, the TKE flux mainly controls the TKE transfer. This result is qualitatively consistent with Costa et al. (2011). The TKE transfer is negative near the surface, while it is positive in the upper part of

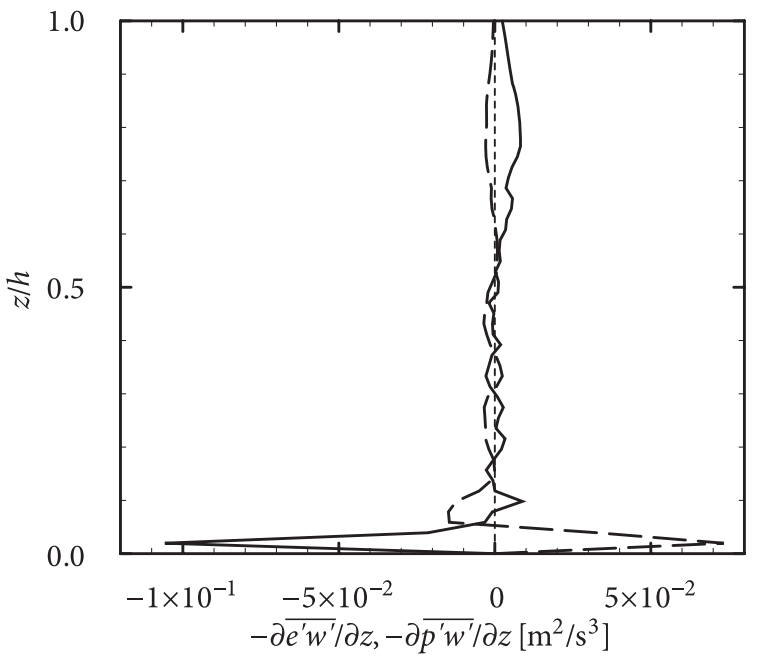

Fig. 7. Vertical profile of the TKE flux term $-\partial \overline{e w^{\prime}} / \partial z$ (solid line) and the pressure-velocity correlation term $-\partial \overline{p^{\prime} w^{\prime}} / \partial z$ (dashed line). The result for a $U=4 \mathrm{~m} \mathrm{~s}^{-1}, \Gamma=$ $8 \mathrm{~K} \mathrm{~m}^{-1}$ case (same as Fig. 5) is presented.

the boundary layer: $0.7<z / h<1$. This vertical distribution corresponds to an upward TKE transfer, which plays a role in supplying TKE to the top of the boundary layer on average. In other words, the TKE transfer term works as downgradient diffusion of TKE, because TKE monotonically decreases with height (not shown).

The TKE transfer term has frequently been parameterized as diffusion of the TKE (e.g., Mellor and Yamada 1982):

$$
\frac{\partial}{\partial z}\left(\overline{e w^{\prime}}+\overline{p^{\prime} w^{\prime}}\right)=-\frac{\partial}{\partial z}\left(K_{e} \frac{\partial \bar{e}}{\partial z}\right)
$$

Here $K_{e}$ represents the eddy viscosity coefficient for TKE. The result in Fig. 7 appears to justify this concept. Figure 8 indicates the diffusion coefficient of TKE calculated by Eq. (6). The diffusion coefficient $K_{e}$ is positive in the entire boundary layer for all ensemble members. This result supports the assumption of downgradient diffusion. This ensemble mean profile has an almost constant value of $4.5 \times 10^{-3} \mathrm{~m}^{2} \mathrm{~s}^{-1}$ in the middle of the boundary layer $0.3<z / h<0.7$, while the spread among the ensemble members is magnified in this region. It increases linearly in the lower layer $z / h$ $<0.3$ and decreases in the top of the layer $0.7<z / h<$ 1 . The mixing length should be $\kappa z$ ( $\kappa$ : the Kármán constant) near a ground surface, which is derived from the logarithmic velocity profile in the constant flux layer, and it becomes smaller at the top of the boundary 


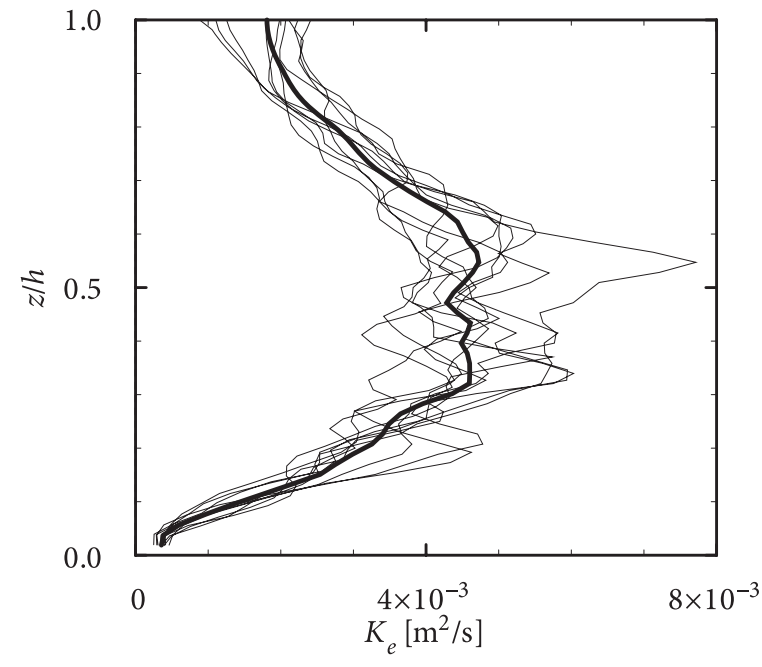

Fig. 8. Vertical profile of the diffusion coefficient associated with the TKE averaged over $t=9.5-10.5 \mathrm{~s}$ for a $U=4 \mathrm{~m} \mathrm{~s}^{-1}, \Gamma=8 \mathrm{~K} \mathrm{~m}^{-1}$ case. The thick line denotes an ensemble mean of ten ensemble members, and the thin line indicates the profile of each ensemble member.

layer because of suppressed vertical mixing in strong stratification. This result suggests that the vertical profiles observed in Fig. 8 could be parameterized in terms of the theory of mixing length.

\section{Concluding remarks}

Wind tunnel experiments and LES for stable stratification cases are performed to investigate Flux Richardson number Rf and turbulent Prandtl number $\mathrm{Pr}$ in terms of dependence on gradient Richardson number Ri. In the present analysis, $\mathrm{Rf}$ and $\mathrm{Pr}^{-1}$ are obtained as a function of $\mathrm{Ri}$ when $10^{-3}<\mathrm{Ri}<5$ using independent samples for the shared variables contained in these nondimensional numbers to avoid the self-correlation problem. Avoiding self-correlation enables us to confirm the appropriateness of the obtained relationship by the theoretical expression $\operatorname{Pr}^{-1}=\mathrm{Rf} / \mathrm{Ri}$. Our result indicates that Rf can exceed unity in strong stratification, in contrast with those of the previous studies in which Rf has an upper limit less than unity. This result suggests that a local balance of TKE cannot be realized because TKE production is negative when $\mathrm{Rf}>1$. It should be noted that our experiments covered a developing boundary layer rather than a stationary one, and the discrepancy from the previous studies associated with the upper value of $\mathrm{Rf}$ might be attributed to characteristics of a developing boundary layer. The assumption of the local balance of TKE cannot be appropriate for a closure model, because a developing boundary layer is not a particular case, but is usually observed.

Furthermore, we analyze the TKE budget in the LES to discuss the TKE balance in the region with negative TKE production. The production and dissipation terms are dominant and cancel each other when $z / h<0.7$, while contribution of the TKE transfer becomes relatively more important than the production and dissipation terms in the top of the boundary layer $(z / h>$ 0.8 ) because these terms are diminished in the upper boundary layer. The TKE is transferred upward and supplied to the top of the boundary layer through the vertical flux of the TKE, while the pressure-velocity correlation term weakens contribution of the TKE flux. This TKE supply causes increased TKE, even though Rf $>1$, corresponding to negative production. Therefore, the assumption of local energy balance is not reasonable in this case; the effects of TKE transfer must be taken into account. The TKE transfer term is well represented as a form of TKE diffusion Eq. (6), which is often assumed in traditional closure models. Our results indicate that the diffusion coefficient $K_{e}$ for TKE is always positive and is qualitatively consistent with the mixing length theory. Further research is required to investigate an appropriate formulation to determine the diffusion coefficient $K_{e}$.

\section{Acknowledgments}

This study is partly supported by JSPS Grant-in-Aid for Scientific Research. All figures are drawn with the GFD-DENNOU Library (SGKS Group 1995). The authors would like to thank two anonymous reviewers for their comments and suggestions, which helped improving the manuscript.

\section{References}

Anderson, P. S., 2009: Measurement of Prandtl number as a function of Richardson number avoiding selfcorrelation. Bound.-Layer Meteor., 131, 345-362.

Baas, P., G. J. Steenveld, B. J. H. van de Wiel, and A. A. M. Holtslag, 2006: Exploring self-correlation in fluxgradient relationships for stably stratified conditions. $J$. Atmos. Sci., 63, 3045-3054.

Businger, J. A., J. C. Wungaard, Y. Izumi, and E. F. Bradley, 1971: Fluxprofile relationships in the atmospheric surface layer. J. Atmos. Sci., 28, 181-189.

Canuto, V. M., Y. Cheng, A. M. Howard, and I. N. Esau, 2008: Stably stratified flows: A model with no Ri(cr). J. Atmos. Sci., 65, 2437-2447.

Costa, F. D., O. C. Acevedo, J. C. M. Mombach, and G. A. Degrazia, 2011: A simplified model for intermittent turbulence in the nocturnal boundary layer. J. Atmos. 
Sci., 68, 1714-1729.

Deardorff, J. W., 1980: Stratocumulus-capped mixed layers derived from a three-dimensional model. Bound.-Layer Meteor., 18, 495-527.

Derbyshire, S. H., 1999: Stable boundary-layer modelling: established appeoaches and beyond. Bound.-Layer Meteor., 90, 423-446.

Esau, I. N., and A. A. Grachev, 2007: Turbulent Prandtl number in stably stratified atmospheric boundary layer: Intercomparison between LES and SHEBA data. $e$ WindEng, 6, 1-17.

Galperin, B., S. Sukoriansky, and P. S. Anderson, 2007: On the critical Richardson number in stably stratified turbulence. Atmos. Sci. Lett., 8, 65-69.

Germano, M., U. Piomelli, P. Moin, and W. H. Cabot, 1991: A dynamic subgrid-scale eddy viscosity model. Phys. Fluids, A3, 1760-1765.

Grachev, A. A., E. L. Andreas, C. W. Fairall, P. S. Guest, and P. O. G. Persson, 2007: On the turbulent Prandtl number in the stable atmospheric boundary layer. Bound.-Layer Meteor., 125, 329-341.

Kitamura, Y., 2010a: Modifications to the Mellor-YamadaNakanishi-Niino (MYNN) model for the stable stratification case. J. Meteor. Soc. Japan, 88, 857-864.

Kitamura, Y., 2010b: Self-consistency validation of subgrid scale parameterization schemes in a large-eddy simulation. J. Meteor. Soc. Japan, 88, 813-825.

Kondo, J., O. Kanechika, and N. Yasuda, 1978: Heat and momentum transfers under strong stability in the atmospheric surface layer. J. Atmos. Sci., 35, 10121021.

Kosović, B., and J. A. Curry, 2000: A large eddy simulation study of a quasisteady, stably stratified atmospheric boundary layer. J. Atmos. Sci., 57, 1052-1068.

Lilly, D. K., 1992: A proposed modification of the germano subgrid-scale closure method. Phys. Fluids, A4, 633635.

Louis, J. F., M. Tiedtke, and J. F. Geleyn, 1982: A short history of the operational PBL-parameterization at ECMWF. Proc. Workshop on Planetary Boundary Layer Parameterization, 59-79.
Mauritsen, T., G. Svensson, S. S. Zilitinkevich, I. Esau, L. Enger, and B. Grisogono, 2007: A total turbulent energy closure model for neutrally and stably stratified atmospheric boundary layers. J. Atmos. Sci., 64, 41134126.

Mellor, G. L., and T. Yamada, 1982: Development of a turbulence closure model for geophysical fluid problems. Rev. Geophys. Space Phys., 20, 851-875.

Metz, W., 1991: Optimal relationship of large-scale flow patterns and the barotropic feedback due to highfrequency eddies. J. Atmos. Sci., 48, 1141-1159.

Monti, P., H. J. S. Fernando, M. Princevac, W. C. Chan, T. A. Kowalewski, and E. R. Pardyjak, 2002: Observations of flow and turbulence in the nocturnal boundary layer over a slope. J. Atmos. Sci., 59, 2513-2534.

Strang, E. J., and H. J. S. Fernando, 2001: Vertical mixing and transports through a stratified shear layer. J. Phys. Oceanogr., 31, 2026-2048.

Tsuji, T., Y. Nagano, and M. Tagawa, 1992: Frequency response and instantaneous temperature profile of coldwire sensors for fluid temperature fluctuation measurements. Experiments in Fluids, 13, 171-178.

Uttal, T., J. A. Curry, M. G. Mcphee, D. K. Perovich, R. E. Moritz, J. A. Maslanik, P. S. Guest, H. L. Stern, J. A. Moore, R. Turenne, A. Heiberg, M. C. Serreze, D. P. Wylie, O. G. Persson, C. A. Paulson, C. Halle, J. H. Morison, P. A. Wheeler, A. Makshtas, H. Welch, M. D. Shupe, J. M. Intrieri, K. Stamnes, R. W. Lindsey, R. Pinkel, W. S. Pegau, T. P. Stanton, and T. C. Grenfeld, 2002: Surface heat budget of the arctic ocean. Bull. Amer. Meteor. Soc., 83, 255-276.

Zilitinkevich, S. S., T. Elperin, N. Kleeorin, and I. Rogachevskii, 2007: Energy- and flux-budget (EFB) turbulence closure model for stably stratified flows. partI: steady-state, homogeneous regimes. Bound.Layer Meteor., 125, 167-191.

Zilitinkevich, S. S., T. Elperin, N. Kleeorin, I. Rogachevskii, I. Esau, T. Mauritsene, and M. W. Miles, 2008: Turbulence energetics in stably stratified geophysical flows: Strong and weak mixing regimes. Quart. J. Roy. Meteor. Soc., 134, 793-799. 